\title{
Boolean constant degree functions on the slice are juntas
}

\author{
Yuval Filmus*, Ferdinand Ihringer ${ }^{\dagger}$
}

July 23, 2019

\begin{abstract}
We show that a Boolean degree $d$ function on the "slice" $\left(\begin{array}{c}{[n]} \\ k\end{array}\right) \triangleq\left\{\left(x_{1}, \ldots, x_{n}\right) \in\right.$ $\left.\{0,1\}: \sum_{i=1}^{n} x_{i}=k\right\}$ is a junta (depends on a constant number $\gamma(d)$ of coordinates), assuming that $k, n-k$ are large enough. This generalizes a classical result of Nisan and Szegedy on the hypercube $\{0,1\}^{n}$. Moreover, we show that the maximum number of coordinates that a Boolean degree $d$ function can depend on is the same on the slice and on the hypercube.
\end{abstract}

\section{Introduction}

Nisan and Szegedy [14] showed that a Boolean degree $d$ function on the hypercube $\{0,1\}^{n}$ depends on at most $d 2^{d-1}$ coordinates, and described a Boolean degree $d$ function which depends on $\Omega\left(2^{d}\right)$ coordinates. The upper bound has recently been improved to $O\left(2^{d}\right)$ by Chiarelli, P. Hatami and Saks [1], who have also improved the hidden constant in the lower bound $\Omega\left(2^{d}\right)$; the hidden constant in the upper bound has subsequently been improved by Wellens [16]. Let us denote the optimal bound by $\gamma(d)$. The goal of this paper is to generalize this result to the slice (or level) $\left(\begin{array}{c}{[n]} \\ k\end{array}\right)$ or Johnson scheme $J(n, k)$, which consists of all points in the hypercube having Hamming weight $k$ :

Theorem 1.1. There exists a constant $C$ such that the following holds. If $C^{d} \leq k \leq$ $n-C^{d}$ and $f:\left(\begin{array}{c}{[n]} \\ k\end{array}\right) \rightarrow\{0,1\}$ has degree $d$, then $f$ depends on at most $\gamma(d)$ coordinates.

(We explain in Section 2 what degree $d$ means for functions on the hypercube and on the slice.)

Filmus et al. [8] proved a version of Theorem 1.1 (with a non-optimal bound on the number of points) when $k / n$ is bounded away from 0,1 , but their bound deteriorates as $k / n$ gets closer to 0,1 . We use their result (which we reproduce here, to keep the proof self-contained) to bootstrap our own inductive argument.

The case $d=1$ is much easier. The following folklore result is proved formally in [7]:

\footnotetext{
*Technion - Israel Institute of Technology, Haifa, Israel. Taub Fellow — supported by the Taub Foundations. This research was funded by ISF grant 1337/16.

$\dagger$ Department of Mathematics: Analysis, Logic and Discrete Mathematics, Ghent University, Belgium. Einstein Institute of Mathematics, Hebrew University of Jerusalem, Israel. Supported by ERC advanced grant 320924. The author is supported by a postdoctoral fellowship of the Research Foundation Flanders (FWO).
} 
Theorem 1.2. If $2 \leq k \leq n-2$ and $f:\left(\begin{array}{c}{[n]} \\ k\end{array}\right) \rightarrow\{0,1\}$ has degree 1 , then $f$ depends on at most one coordinate.

The bounds on $k$ in this theorem are optimal, since every function on $\left(\begin{array}{c}{[n]} \\ 1\end{array}\right)$ and on $\left(\begin{array}{c}{[n]} \\ n-1\end{array}\right)$ has degree 1 . In contrast, the bounds on $k$ in Theorem 1.1 are probably not optimal, an issue we discuss in Section 4.

Let us close this introduction by mentioning a recent result of Keller and Klein [11], which studies Boolean functions on $\left(\begin{array}{c}{[n]} \\ k\end{array}\right)$ which are $\epsilon$-close to being degree $d$, where distance is measured using the squared $L_{2}$ norm. Assuming that $k \leq n / 2$, their result states that if $\epsilon<(k / n)^{O(d)}$ then $f$ is $O(\epsilon)$-close to a junta.

\section{Preliminaries}

In this paper, we discuss Boolean functions, which are 0,1-valued functions, on two different domains: the hypercube and the slice. We will use the notation $[n]:=\{1, \ldots, n\}$. A degree $d$ function (in a context in which degree is defined) is a function of degree at most $d$ (over the reals).

The hypercube. The $n$-dimensional hypercube is the domain $\{0,1\}^{n}$. Every function on the hypercube can be represented uniquely as a real multilinear polynomial in the $n$ input arguments $x_{1}, \ldots, x_{n}$. The degree of a function on the hypercube is the degree of this polynomial. Alternatively, the degree of a function on the hypercube is the minimum degree of a real polynomial in $x_{1}, \ldots, x_{n}$ which agrees with the function on all points of the hypercube. A function on the hypercube is an $m$-junta if it depends on at most $m$ inputs, that is, if there exists a set $I$ of $m$ inputs such that $f(x)=f(y)$ as long as $x_{i}=y_{i}$ for all $i \in I$; we also say that $f$ is an $I$-junta. For more information on functions on the hypercube from this perspective, consult O'Donnell's monograph [15].

The slice. Let $0 \leq k \leq n$. The slice $\left(\begin{array}{c}{[n]} \\ k\end{array}\right)$ is the subset of $\{0,1\}^{n}$ consisting of all vectors having Hamming weight $k$, or in other words, the $k$ th level of the Boolean lattice of subsets of $[n]$. The slice appears naturally in combinatorics, coding theory, and elsewhere, and is known to algebraic combinatorialists as the Johnson scheme $J(n, k)$.

Every function on the slice can be represented uniquely as a real multilinear polynomial $P$ in the $n$ input arguments $x_{1}, \ldots, x_{n}$ of degree at most $\min (k, n-k)$ which satisfies $\sum_{i=1}^{n} \frac{\partial P}{\partial x_{i}}=0$ (the latter condition is known as harmonicity). The degree of a function on the slice is the degree of this polynomial. Alternatively, the degree of a function on the slice is the minimum degree of a real polynomial in $x_{1}, \ldots, x_{n}$ (not necessarily multilinear or harmonic) which agrees with the function on all points of the slice.

Note that a degree $d$ function on the slice $\left(\begin{array}{c}{[n]} \\ k\end{array}\right)$ is also a degree $d$ function on the slice $\left(\begin{array}{c}{[n]} \\ n-k\end{array}\right)$ and vice versa. The isomorphism between $\left(\begin{array}{c}{[n]} \\ k\end{array}\right)$ consists of switching zeroes and ones or, in other words, of replacing each set by its complement. Due to this we can assume that $k \leq n / 2$ without loss of generality whenever it is suitable for our calculations. This also explains why all our bounds on $k$ are symmetric throughout the document (as in Theorem 1.1).

A function $f$ on the slice is an m-junta if there exist a function $g:\{0,1\}^{m} \rightarrow\{0,1\}$ and $m$ indices $i_{1}<\ldots<i_{m}$ such that $f(x)=g\left(\left.x\right|_{i_{1}, \ldots, i_{m}}\right)$, where $\left.x\right|_{i_{1}, \ldots, i_{m}}=x_{i_{1}}, \ldots, x_{i_{m}}$. 
Alternatively, $f$ is an $m$-junta if there exists a set $I$ of $m$ coordinates such that $f$ is invariant under permutation of the coordinates in $[n] \backslash I$; we also say that $f$ is an $I$ junta. Note that the set $I$ is not defined uniquely (in contrast to the hypercube case): for example, $f=\sum_{i \in I} x_{i}$ is both an $I$-junta and an $[n] \backslash I$-junta.

The $p$ th norm of $f$ is given by $\|f\|_{p}=\mathbb{E}\left[|f|^{p}\right]^{1 / p}$, where the expectation is over a uniform point in the slice. In particular, $\|f\|_{2}^{2}=\mathbb{E}\left[f^{2}\right]$.

Let $f$ be a Boolean function on the slice $\left(\begin{array}{c}{[n]} \\ k\end{array}\right)$, and let $P$ be its unique harmonic multilinear polynomial representation. The $d$ th level of $f$, denoted $f=d$, is the homogeneous degree $d$ part of $P$ (the sum of all degree $d$ monomials with their coefficients). The different levels are orthogonal: $\mathbb{E}\left[f^{=d} f^{=e}\right]=0$ if $d \neq e$. Orthogonality of the different levels implies that

$$
\|f\|_{2}^{2}=\sum_{d=0}^{\operatorname{deg} f}\left\|f^{=d}\right\|_{2}^{2} .
$$

Let $f$ be a Boolean function on the slice $\left(\begin{array}{c}{[n]} \\ k\end{array}\right)$, and let $i, j \in[n]$. We define $f^{(i j)}$ to be the function given by $f^{(i j)}(x)=f\left(x^{(i j)}\right)$, where $x^{(i j)}$ is obtained from $x$ by switching $x_{i}$ and $x_{j}$. The $(i, j)$ th influence of $f$ is $\operatorname{Inf}_{i j}[f]=\frac{1}{4} \operatorname{Pr}\left[f(x) \neq f\left(x^{(i j)}\right)\right]$, where $x$ is chosen uniformly at random over the slice. An equivalent formula is $\operatorname{Inf}_{i j}[f]=\frac{1}{4} \mathbb{E}[(f-$ $\left.\left.f^{(i j)}\right)^{2}\right]$. Clearly $\operatorname{Inf}_{i j}[f]=0$ if and only if $f=f^{(i j)}$. The total influence of $f$ is $\operatorname{Inf}[f]=$ $\frac{1}{n} \sum_{1 \leq i<j \leq n} \operatorname{Inf}_{i j}[f]$. It is given by the formula [5, Lemma 27]

$$
\operatorname{Inf}[f]=\sum_{d=0}^{\operatorname{deg} f} \frac{d(n+1-d)}{n}\left\|f^{=d}\right\|_{2}^{2} .
$$

For a parameter $\rho \in(0,1]$, the noise operator $T_{\rho}$, mapping functions on the slice to functions on the slice, is defined by

$$
T_{\rho} f=\sum_{d=0}^{\operatorname{deg} f} \rho^{d(1-(d-1) / n)} f^{=d} .
$$

Alternatively [5, Lemma 27], $\left(T_{\rho} f\right)(x)$ is the expected value of $f(y)$, where $y$ is chosen by applying $N \sim \operatorname{Po}\left(\frac{n-1}{2} \log (1 / \rho)\right)$ random transpositions to $x$. Lee and Yau [12] proved a $\log$ Sobolev inequality, which together with classical results of Diaconis and SaloffCoste [2] implies that the following hypercontractive inequality holds for some constant $C_{H}>0$ :

$$
\left\|T_{\rho} f\right\|_{2} \leq\|f\|_{4 / 3}, \quad \rho=\left(\frac{2 k(n-k)}{n(n-1)}\right)^{C_{H}} .
$$

For more information on functions on the slice, consult $[5,9]$.

\section{Main theorem}

For the rest of this section, we fix an integer $d \geq 1$. Our goal is to prove Theorem 1.1 for this value of $d$. We will use the phrase universal constant to refer to a constant independent of $d$.

The strategy of the proof is to proceed in three steps: 
1. Bootstrapping: Every Boolean degree $d$ function on $\left(\begin{array}{c}{[2 n]} \\ n\end{array}\right)$ is a $K^{d}$-junta.

2. Induction: If every Boolean degree $d$ function on $\left(\begin{array}{c}{[n]} \\ k\end{array}\right)$ is an $M$-junta, then the same holds for $\left(\begin{array}{c}{[n+1]} \\ k\end{array}\right)$ and $\left(\begin{array}{c}{[n+1]} \\ k+1\end{array}\right)$ (under certain conditions).

3. Culmination: If a Boolean degree $d$ function on $\left(\begin{array}{c}{[n]} \\ k\end{array}\right)$ is an $L$-junta but not an $(L-1)$ junta, then (under certain conditions) there exists a Boolean degree $d$ function on the hypercube depending on $L$ coordinates.

We also show a converse to the last step: given a Boolean degree $d$ function on the hypercube depending on $L$ coordinates, we show how to construct Boolean degree $d$ functions on large enough slices that are $L$-juntas but not $(L-1)$-juntas.

\subsection{Bootstrapping}

We bootstrap our approach by proving that every Boolean degree $d$ function on $\left(\begin{array}{c}{[2 n]} \\ n\end{array}\right)$ is a junta. The proof is a simple application of hypercontractivity, and already appears in [8]. We reproduce a simplified version here in order to make the paper self-contained.

The main idea behind the proof is to obtain a dichotomy on the influences of the function.

Lemma 3.1. There exists a universal constant $\alpha$ such that all non-zero influences of a Boolean degree $d$ function on $\left(\begin{array}{c}{[2 n]} \\ n\end{array}\right)$ are at least $\alpha^{d}$.

Proof. Let $f$ be a Boolean degree $d$ function on $\left(\begin{array}{c}{[2 n]} \\ n\end{array}\right)$. Given $i, j \in[n]$, consider the function $f_{i j}=\left(f-f^{(i j)}\right) / 2$, related to the $(i, j)$ th influence of $f$ by $\operatorname{Inf}_{i j}[f]=\left\|f_{i j}\right\|_{2}^{2}$. Since $f$ is 0,1 -valued, $f_{i j}$ is $0, \pm 1$-valued. Since $f$ has degree $d$, it follows that $f_{i j}$ can be written as a degree $d$ polynomial, and so has degree at most $d$. Hypercontractivity (3) implies that for some universal constant $\rho$, we have

$$
\left\|T_{\rho} f_{i j}\right\|_{2} \leq\left\|f_{i j}\right\|_{4 / 3}
$$

We can estimate the left-hand side of (4) using (2):

$$
\left\|T_{\rho} f_{i j}\right\|_{2}^{2}=\sum_{e=0}^{d} \rho^{2 e(1-(e-1) / n)}\left\|f_{i j}^{=e}\right\|_{2}^{2} \geq \rho^{2 d} \sum_{e=0}^{d}\left\|f_{i j}^{=e}\right\|_{2}^{2}=\rho^{2 d}\left\|f_{i j}\right\|_{2}^{2}=\rho^{2 d} \operatorname{Inf}_{i j}[f] .
$$

We can calculate the right-hand side of (4) using the fact that $f_{i j}$ is $0, \pm 1$-valued:

$$
\left\|f_{i j}\right\|_{4 / 3}^{4 / 3}=\mathbb{E}\left[\left|f_{i j}\right|^{4 / 3}\right]=\mathbb{E}\left[\left|f_{i j}\right|^{2}\right]=\left\|f_{i j}\right\|_{2}^{2}=\operatorname{Inf}_{i j}[f] .
$$

Combining the estimates on both sides of (4), we conclude that

$$
\rho^{2 d} \operatorname{Inf}_{i j}[f] \leq \operatorname{Inf}_{i j}[f]^{3 / 2} .
$$

Hence either $\operatorname{Inf}_{i j}[f]=0 \operatorname{or}_{\operatorname{Inf}_{i j}}[f] \geq \rho^{4 d}$.

The next step is to prove a degenerate triangle inequality (for the non-degenerate version, see Wimmer [17, Lemma 5.4] and Filmus [5, Lemma 5.1]). 
Lemma 3.2. Let $f$ be a function on a slice. If $\operatorname{Inf}_{i k}[f]=\operatorname{Inf}_{j k}[f]=0$ then $\operatorname{Inf}_{i j}[f]=0$. Proof. If $\operatorname{Inf}_{i k}[f]=\operatorname{Inf}_{j k}[f]=0$ then $f(x)=f\left(x^{(i k)}\right)=f\left(x^{(j k)}\right)$, and so $f(x)=$ $f\left(x^{(i k)(j k)(i k)}\right)=f\left(x^{(i j)}\right)$. It follows that $\operatorname{Inf}_{i j}[f]=0$.

To complete the proof, we use formula (1), which implies that $\operatorname{Inf}[f] \leq d$ for any Boolean degree $d$ function $f$, together with an idea of Wimmer [17, Proposition 5.3].

Lemma 3.3. There exists a universal constant $K>1$ such that for $n \geq 2$, every Boolean degree d function on $\left(\begin{array}{c}{[2 n]} \\ n\end{array}\right)$ is a $K^{d}$-junta.

Proof. Let $f$ be a Boolean degree $d$ function on $\left(\begin{array}{c}{[2 n]} \\ n\end{array}\right)$. Construct a graph $G$ on the vertex set $[2 n]$ by connecting two vertices $i, j$ if $\operatorname{Inf}_{i j}[f] \geq \alpha^{d}$, where $\alpha$ is the constant from Lemma 3.1. Let $M$ be a maximal matching in $G$. It is well-known that the $2|M|$ vertices of $M$ form a vertex cover $V$, that is, any edge of $G$ touches one of these vertices. Therefore if $i, j \notin V$ then $\operatorname{Inf}_{i j}[f]<\alpha^{d}$, and so $\operatorname{Inf}_{i j}[f]=0$ according to Lemma 3.1. In other words, $f$ is a $V$-junta. It remains to bound the size of $V$.

Let $(i, j)$ be any edge of $M$, and let $k$ be any other vertex. Lemma 3.2 shows that either $\operatorname{Inf}_{i k}[f] \neq 0$ or $\operatorname{Inf}_{j k}[f] \neq 0$, and so either $(i, k)$ or $(j, k)$ is an edge of $G$, according to Lemma 3.1. It follows that $G$ contains at least $|M|(2 n-2) / 2$ edges (we divided by two since some edges could be counted twice). Therefore $n \operatorname{Inf}[f]=\sum_{1 \leq i<j \leq n} \operatorname{Inf}_{i j}[f] \geq$ $\alpha^{d}|M|(n-1)$. On the other hand, (1) shows that

$$
\operatorname{Inf}[f]=\sum_{e=0}^{d} \frac{e(n+1-e)}{n}\left\|f^{=e}\right\|_{2}^{2} \leq d \sum_{e=0}^{d}\left\|f^{=e}\right\|_{2}^{2}=d\|f\|_{2}^{2} \leq d .
$$

It follows that

$$
|V|=2|M| \leq 2 \frac{n}{n-1} d(1 / \alpha)^{d} \leq(8 / \alpha)^{d}
$$

\subsection{Induction}

The heart of the proof is an inductive argument which shows that if Theorem 1.1 holds (with a non-optimal bound on the size of the junta) for the slice $\left(\begin{array}{c}{[n]} \\ k\end{array}\right)$, then it also holds for the slices $\left(\begin{array}{c}{[n+1]} \\ k\end{array}\right)$ and $\left(\begin{array}{c}{[n+1]} \\ k+1\end{array}\right)$, assuming that $n$ is large enough and that $k$ is not too close to 0 or $n$. Given a Boolean degree $d$ function $f$ on $\left(\begin{array}{c}{[n+1]} \\ k\end{array}\right)$ or $\left(\begin{array}{c}{[n+1]} \\ k+1\end{array}\right)$, the idea is to consider restrictions of $f$ obtained by fixing one of the coordinates.

Lemma 3.4. Suppose that every Boolean degree d function on $\left(\begin{array}{c}{[n]} \\ k\end{array}\right)$ is an M-junta, where $M \geq 1$.

Let $f$ be a Boolean degree $d$ function on $\left(\begin{array}{c}n+1] \\ k+b\end{array}\right)$, where $b \in\{0,1\}$. For each $i \in[n+1]$, let $f_{i}$ be the restriction of $f$ to vectors satisfying $x_{i}=b$. Then for each $i \in[n+1]$ there exists a set $S_{i} \subseteq[n+1] \backslash\{i\}$ of size at most $M$ and a function $g_{i}:\{0,1\}^{S_{i}} \rightarrow\{0,1\}$, depending on all inputs, such that $f_{i}(x)=g_{i}\left(\left.x\right|_{S_{i}}\right)$.

Proof. Choose $i \in[n+1]$. The domain of $f_{i}$ is isomorphic to $\left(\begin{array}{c}{[n]} \\ k\end{array}\right)$. Moreover, since $f$ can be represented as a polynomial of degree $d$, so can $f_{i}$, hence $\operatorname{deg} f_{i} \leq d$. By assumption, $f_{i}$ is an $M$-junta, and so $f_{i}(x)=h_{i}\left(\left.x\right|_{T_{i}}\right)$ for some set $T_{i}$ of $M$ indices and some function $h_{i}:\{0,1\}^{T_{i}} \rightarrow\{0,1\}$. Let $S_{i} \subseteq T_{i}$ be the set of inputs that $h_{i}$ depends on. Then there exists a function $g_{i}:\{0,1\}^{S_{i}} \rightarrow\{0,1\}$ such that $g_{i}\left(\left.x\right|_{S_{i}}\right)=h_{i}\left(\left.x\right|_{T_{i}}\right)$, completing the proof. 
Each of the sets $S_{i}$ individually contains at most $M$ indices. We now show that in fact they contain at most $M$ indices in total.

Lemma 3.5. Under the assumptions of Lemma 3.4, suppose further that $n \geq(M+1)^{3}$ and $M+2 \leq k \leq n+1-(M+2)$.

The union of any $M+1$ of the sets $S_{i}$ contains at most $M$ indices.

Proof. We can assume, without loss of generality, that the sets in question are $S_{1}, \ldots, S_{M+1}$. Denote their union by $A$, and let $B=A \cup\{1, \ldots, M+1\}$. Since $|B| \leq(M+1)^{2} \leq n$, there exists a point $r \in[n+1] \backslash B$. We proceed by bounding the number of unordered pairs of distinct indices $i, j \in[n+1] \backslash\{r\}$ such that $f_{r}=f_{r}^{(i j)}$, which we denote by $N$. Since $f_{r}$ is an $M$-junta, we know that $N \geq\left(\begin{array}{c}n-M \\ 2\end{array}\right)$. We will now obtain an upper bound on $N$ in terms of $|A|$ and $|B|$.

Let $1 \leq \ell \leq M+1$, and suppose that $i \in S_{\ell}$ and $j \notin S_{\ell} \cup\{\ell, r\}$. We claim that $f_{r}^{(i j)} \neq f_{r}$. Indeed, since $g_{\ell}$ depends on all inputs, there are two inputs $y, z$ to $g_{\ell}$, differing only on the $i$ th coordinate, say $y_{i}=b$ and $z_{i}=1-b$, such that $g_{\ell}(y) \neq g_{\ell}(z)$. Since $M+2 \leq k \leq n+1-(M+2)$, we can extend $y$ to an input $x$ to $f$ satisfying additionally the constraints $x_{\ell}=x_{r}=b$ and $x_{j}=1-b$. Since $x_{\ell}=x_{r}=b$, the input $x$ is in the common domain of $f_{\ell}$ and $f_{r}$. Notice that $f_{r}(x)=f_{\ell}(x)=g_{\ell}(y)$, whereas $f_{r}\left(x^{(i j)}\right)=f_{\ell}\left(x^{(i j)}\right)=g_{\ell}(z)$, since $x_{i}=y_{i}=b$ whereas $x_{j}=1-b$. By construction $g_{\ell}(y) \neq g_{\ell}(z)$, and so $f_{r} \neq f_{r}^{(i j)}$.

The preceding argument shows that if $i \in A$ and $j \notin B \cup\{r\}$ then $f_{r} \neq f_{r}^{(i j)}$. Therefore $\left(\begin{array}{l}n \\ 2\end{array}\right)-N \geq|A|(n-|B|) \geq|A|\left(n-(M+1)^{2}\right)$. Combining this with the lower bound $N \geq\left(\begin{array}{c}n-M \\ 2\end{array}\right)$, we deduce that

$$
|A|\left(n-(M+1)^{2}\right) \leq\left(\begin{array}{l}
n \\
2
\end{array}\right)-\left(\begin{array}{c}
n-M \\
2
\end{array}\right)=\frac{M(2 n-M-1)}{2} .
$$

Rearrangement shows that

$$
|A| \leq \frac{M(2 n-M-1)}{2\left(n-(M+1)^{2}\right)}=\left(1+\frac{(M+1)(2 M+1)}{2 n-2(M+1)^{2}}\right) M
$$

When $n>\left(M^{2}+(3 / 2) M+1\right)(M+1)$, we have $\frac{(M+1)(2 M+1)}{2 n-2(M+1)^{2}}<\frac{1}{M}$, and so $|A|<M+1$. We conclude that when $n \geq(M+1)^{3}$, we have $|A| \leq M$.

Corollary 3.6. Under the assumptions of the preceding lemma, the union of $S_{1}, \ldots, S_{n+1}$ contains at most $M$ indices.

Proof. Suppose that the union contained at least $M+1$ indices $i_{1}, \ldots, i_{M+1}$. Each index $i_{t}$ is contained in some set $S_{j_{t}}$, and in particular the union of $S_{j_{1}}, \ldots, S_{j_{M+1}}$ contains at least $M+1$ indices, contradicting the lemma.

Denoting the union of all $S_{i}$ by $S$, it remains to show that $f$ is an $S$-junta.

Lemma 3.7. Suppose that every Boolean degree d function on $\left(\begin{array}{c}{[n]} \\ k\end{array}\right)$ is an $M$-junta, where $M \geq 1$; that $n \geq(M+1)^{3}$; and that $M+2 \leq k \leq n+1-(M+2)$.

Any Boolean degree d function on $\left(\begin{array}{c}{[n+1]} \\ k\end{array}\right)$ or on $\left(\begin{array}{c}{[n+1]} \\ k+1\end{array}\right)$ is an $M$-junta. 
Proof. Let $b, f_{i}, g_{i}, S_{i}$ be defined as in Lemma 3.4, and let $S$ denote the union of $S_{1}, \ldots, S_{n+1}$. Corollary 3.6 shows that $|S| \leq M$. Since $n \geq(M+1)^{3}$, it follows that there exists an index $r \in[n+1] \backslash S$. We will show that $f(x)=g_{r}\left(\left.x\right|_{S_{r}}\right)$, and so $f$ is an $M$-junta.

Consider any input $x$ to $f$. If $x_{r}=b$ then $x$ is in the domain of $f_{r}$, and so clearly $f(x)=f_{r}(x)=g_{r}\left(\left.x\right|_{S_{r}}\right)$. Suppose therefore that $x_{r}=1-b$. Since $M+2 \leq k \leq$ $n+1-(M+2)$, there exists a coordinate $s \in[n+1] \backslash S$ such that $x_{s}=b$, putting $x$ in the domain of $f_{s}$. Again since $M+2 \leq k \leq n+1-(M+2)$, there exists a coordinate $t \in[n+1] \backslash(S \cup\{s\})$ such that $x_{t}=b$. Since $x^{(r t)}$ is in the domain of $f_{r}$, we have

$$
f(x)=f_{s}(x)=g_{s}\left(\left.x\right|_{S_{s}}\right)=g_{s}\left(x_{S_{s}}^{(r t)}\right)=f_{s}\left(x^{(r t)}\right)=f_{r}\left(x^{(r t)}\right)=g_{r}\left(\left.x^{(r t)}\right|_{S_{r}}\right)=g_{r}\left(\left.x\right|_{S_{r}}\right) .
$$

\subsection{Culmination}

Combining Lemma 3.3 and Lemma 3.7, we obtain a version of Theorem 1.1 with a suboptimal upper bound on the size of the junta.

Lemma 3.8. There exists a universal constant $C>1$ such that whenever $C^{d} \leq k \leq$ $n-C^{d}$, every Boolean degree d function on $\left(\begin{array}{c}{[n]} \\ k\end{array}\right)$ is a $C^{d}$-junta.

Proof. Let $K$ be the constant from Lemma 3.3, and let $M=K^{d}$. We choose $C:=$ $(K+2)^{3}$.

Let us assume that $k \leq n / 2$ (the proof for $k \geq n / 2$ is very similar). Lemma 3.3 shows that every Boolean degree $d$ function on $\left(\begin{array}{c}{[2 k]} \\ k\end{array}\right)$ is an $M$-junta. If $m \geq 2 k$ then $m \geq 2 k \geq(M+1)^{3}$ and $M+2 \leq k \leq m-(M+2)$. Therefore Lemma 3.7 shows that if every Boolean degree $d$ function on $\left(\begin{array}{c}{[m]} \\ k\end{array}\right)$ is an $M$-junta, then the same holds for $\left(\begin{array}{c}{[m+1]} \\ k\end{array}\right)$. Applying the lemma $n-2 k$ times, we conclude that every Boolean degree $d$ function on $\left(\begin{array}{c}{[n]} \\ k\end{array}\right)$ is a $C^{d}$-junta.

To complete the proof of the theorem, we show how to convert a Boolean degree $d$ function on the slice depending on many coordinates to a Boolean degree $d$ function on the hypercube depending on the same number of coordinates.

Lemma 3.9. Suppose that $f$ is a Boolean degree $d$ function on $\left(\begin{array}{c}{[n]} \\ k\end{array}\right)$ which is an L-junta but not an $(L-1)$-junta, where $L \leq k, n-k$. Then there exists a Boolean degree $d$ function $g$ on $\{0,1\}^{L}$ which depends on all coordinates.

Proof. Without loss of generality, we can assume that $f(x)=g\left(x_{1}, \ldots, x_{L}\right)$ for some Boolean function $g$ on the $L$-dimensional hypercube. Since $f$ is not an $(L-1)$-junta, the function $g$ depends on all coordinates. Since $L \leq k, n-k$, as $x$ goes over all points in $\left(\begin{array}{c}{[n]} \\ k\end{array}\right)$, the vector $x_{1}, \ldots, x_{L}$ goes over all points in $\{0,1\}^{L}$. It remains to show that there is a degree $d$ polynomial agreeing with $g$ on $\{0,1\}^{L}$.

Since $f$ has degree at most $d$, there is a degree $d$ multilinear polynomial $P$ such that $f=P$ for every point in $\left(\begin{array}{c}{[n]} \\ k\end{array}\right)$. If $\pi$ is any permutation of $\{L+1, \ldots, n\}$ then $f=f^{\pi}$, where $f^{\pi}(x)=f\left(x^{\pi}\right)$. Denoting the set of all such permutations by $\Pi$, if we define $Q:=\mathbb{E}_{\pi \in \Pi}\left[P^{\pi}\right]$ then $f=Q$ for every point in $\left(\begin{array}{c}{[n]} \\ k\end{array}\right)$. The polynomial $Q$ is a degree $d$ polynomial which is invariant under permutations from $\Pi$.

For $a_{1}, \ldots, a_{L} \in\{0,1\}^{L}$ summing to $a \leq d$, let $Q_{a_{1}, \ldots, a_{L}}\left(x_{L+1}, \ldots, x_{n}\right)$ be the coefficient of $\prod_{i=1}^{L} x_{i}^{a_{i}}$ in $Q$. This is a degree $d-a$ symmetric polynomial in $x_{L+1}, \ldots, x_{n}$, and so a 
classical result of Minsky and Papert [13] (see also [14, Lemma 3.2]) implies that there exists a degree $d-a$ univariate polynomial $R_{a_{1}, \ldots, a_{L}}$ such that $Q_{a_{1}, \ldots, a_{L}}\left(x_{L+1}, \ldots, x_{n}\right)=$ $R_{a_{1}, \ldots, a_{L}}\left(x_{L+1}+\cdots+x_{n}\right)$ for all $x_{L+1}, \ldots, x_{n} \in\{0,1\}^{n-L}$. Since $x_{L+1}+\cdots+x_{n}=$ $k-x_{1}-\cdots-x_{L}$, it follows that for inputs $x$ in $\left(\begin{array}{c}{[n]} \\ k\end{array}\right)$, we have

$$
f\left(x_{1}, \ldots, x_{n}\right)=\sum_{\substack{a_{1}, \ldots, a_{L} \in\{0,1\} \\ a_{1}+\cdots+a_{L} \leq d}} \prod_{i=1}^{L} x_{i}^{a_{i}} \cdot R_{a_{1}, \ldots, a_{L}}\left(k-x_{1}-\cdots-x_{L}\right) .
$$

The right-hand side is a degree $d$ polynomial in $x_{1}, \ldots, x_{L}$ which agrees with $g$ on $\{0,1\}^{L}$.

Theorem 1.1 immediately follows from combining Lemma 3.8 and Lemma 3.9.

We conclude this section by proving a converse of Lemma 3.10.

Lemma 3.10. Suppose that $g$ is a Boolean degree $d$ function on $\{0,1\}^{L}$ depending on all coordinates. Then for all $n, k$ satisfying $L \leq k \leq n-L$ there exists a Boolean degree $d$ function $f$ on $\left(\begin{array}{c}{[n]} \\ k\end{array}\right)$ which is an L-junta but not an $(L-1)$-junta.

Proof. We define $f\left(x_{1}, \ldots, x_{n}\right)=g\left(x_{1}, \ldots, x_{L}\right)$. Clearly, $f$ is an $L$-junta. Since $g$ has degree at most $d$, there is a polynomial $P$ which agrees with $g$ on all points of $\{0,1\}^{L}$. The same polynomial also agrees with $f$ on all points of $\left(\begin{array}{c}{[n]} \\ k\end{array}\right)$, and so $f$ also has degree at most $d$. It remains to show that $f$ is not an $(L-1)$-junta.

Suppose, for the sake of contradiction, that $f$ were an $(L-1)$-junta. Then there exists a set $S$ of size at most $L-1$ and a Boolean function $h:\{0,1\}^{S} \rightarrow\{0,1\}$ such that $f(x)=h\left(\left.x\right|_{S}\right)$. Since $|S|<L$, there exists some coordinate $i \in\{1, \ldots, L\} \backslash S$. Since $g$ depends on all coordinates, there are two inputs $y, z$ to $g$ differing only in the $i$ th coordinate, say $y_{i}=0$ and $z_{i}=1$, such that $g(y) \neq g(z)$. Since $n \geq 2 L$, there exists a coordinate $j \in[n] \backslash(\{1, \ldots, L\} \cup S)$. Since $L \leq k \leq n-L$, we can extend $y$ to an input $\tilde{y}$ to $f$ such that $x_{j}=1$. The input $\tilde{z}=\tilde{y}^{(i j)}$ extends $z$. Since $i, j \notin S$, the inputs $\tilde{y}, \tilde{z}$ agree on all coordinates in $S$, and so $f(\tilde{y})=h\left(\left.\tilde{y}\right|_{S}\right)=h\left(\left.\tilde{z}\right|_{S}\right)=f(\tilde{z})$. On the other hand, $f(\tilde{y})=g(y) \neq g(z)=f(\tilde{z})$. This contradiction shows that $f$ cannot be an $(L-1)$-junta.

\section{Discussion}

Optimality. Lemma 3.10 shows that the size of the junta in Theorem 1.1 is optimal. However, it is not clear whether the bounds on $k$ are optimal. The theorem fails when $k \leq d$ or $k \geq n-d$, since in these cases every function has degree $d$. This prompts us to define the following two related quantities:

1. $\zeta(d)$ is the minimal value such that every Boolean degree $d$ function on $\left(\begin{array}{c}{[n]} \\ k\end{array}\right)$ is an $O_{d}(1)$-junta whenever $\zeta(d) \leq k \leq n-\zeta(d)$.

2. $\xi(d)$ is the minimal value such that every Boolean degree $d$ function on $\left(\begin{array}{c}{[n]} \\ k\end{array}\right)$ is a $\gamma(d)$-junta whenever $\xi(d) \leq k \leq n-\xi(d)$. 
Clearly $d<\zeta(d) \leq \xi(d)$. We can improve this to $\zeta(d) \geq \eta(d) \geq 2\left\lceil\frac{d+1}{2}\right\rceil$, where $\eta(d)$ is the maximum integer such that there exists a non-constant univariate degree $d$ polynomial $P_{d}$ satisfying $P_{d}(0), \ldots, P_{d}(\eta(d)-1) \in\{0,1\}$. Given such a polynomial $P_{d}$, we can construct a degree $d$ function $f_{d}$ on $\left(\begin{array}{c}{[n]} \\ k\end{array}\right)$ which is not a junta:

$$
f_{d}\left(x_{1}, \ldots, x_{n}\right)=P_{d}\left(x_{1}+\cdots+x_{\lfloor n / 2\rfloor}\right) .
$$

When $k<\eta(d)$, the possible values of $x_{1}+\cdots+x_{\lfloor n / 2\rfloor}$ are such that $f_{d}$ is Boolean. One can check that $f_{d}$ is not an $L$-junta unless $L \geq\lfloor n / 2\rfloor$. The following polynomial shows that $\eta(d) \geq 2\left\lceil\frac{d+1}{2}\right\rceil$ :

$$
P_{d}(\sigma)=\sum_{e=0}^{d}(-1)^{e}\left(\begin{array}{l}
\sigma \\
e
\end{array}\right),
$$

where $\left(\begin{array}{l}\sigma \\ 0\end{array}\right)=1$. While this construction can be improved for specific $d$ (for example, $\eta(7)=9$ and $\eta(12)=16)$, the upper bound $\eta(d) \leq 2 d$ shows that this kind of construction cannot give an exponential lower bound on $\zeta(d)$.

Curiously, essentially the same function appears in [3, Section 7] as an example of a degree $d$ function on the biased hypercube which is almost Boolean but somewhat far from being constant.

Extensions. It would be interesting to extend Theorem 1.1 to other domains. In recent work [7], we explored Boolean degree 1 functions on various domains, including various association schemes and finite groups, and the multislice (consult the work for the appropriate definitions). Inspired by these results, we make the following conjectures:

1. If $f$ is a Boolean degree $d$ function on the Grassmann scheme then there are $O(1)$ points and hyperplanes such that $f(S)$ depends only on which of the points is contained in $S$, and which of the hyperplanes contain $S$. The case $d=1$ has been proved in [7] for $q=2,3,4,5$.

2. If $f$ is a Boolean degree $d$ function on the multislice $M\left(k_{1}, \ldots, k_{m}\right)$ for $k_{1}, \ldots, k_{m} \geq$ $\exp (d)$ then $f$ is a $\gamma_{m}(d)$-junta, where $\gamma_{m}(d)$ is the maximum number of coordinates that a Boolean degree $d$ function on the Hamming scheme $H(n, m)$ can depend on. This conjecture has subsequently been proved in [10], by closely following the argument in this paper.

3. If $f$ is a Boolean degree $d$ function on the symmetric group then it is computed by a constant depth $n$-way decision tree whose internal nodes correspond to queries of the form $\pi(i)$ or $\pi^{-1}(j)$. The case $d=1$ has been proved by Ellis, Friedgut and Pilpel [4]. ${ }^{1}$

We leave it to the reader to show that $\gamma_{m}(d)$ exists. In fact, simple arguments show that $m^{d-1} \leq \gamma_{m}(d) \leq \gamma\left(\left\lceil\log _{2} m\right\rceil d\right)$.

\footnotetext{
${ }^{1}$ Theorem 27 of [4] would imply this conjecture, but unfortunately the result is false [6].
} 


\section{References}

[1] John Chiarelli, Pooya Hatami, and Michael Saks. Tight bound on the number of relevant variables in a bounded degree Boolean function. CoRR, abs/1801.08564, 2018.

[2] Persi Diaconis and Laurent Saloff-Coste. Logarithmic Sobolev inequalities for finite Markov chains. Ann. Appl. Probab., 6(3):695-750, Aug 1996.

[3] Irit Dinur, Yuval Filmus, and Prahladh Harsha. Low degree almost Boolean functions are sparse juntas. CoRR, abs/1711.09428, 2017.

[4] David Ellis, Ehud Friedgut, and Haran Pilpel. Intersecting families of permutations. J. Amer. Math. Soc., 24:649-682, 2011.

[5] Yuval Filmus. Orthogonal basis for functions over a slice of the Boolean hypercube. Electronic J. Comb., 23(1):P1.23, 2016.

[6] Yuval Filmus. A comment on Intersecting Families of Permutations. CoRR, abs/1706.10146, 2017.

[7] Yuval Filmus and Ferdinand Ihringer. Boolean degree 1 functions on some classical association schemes. Journal of Combinatorial Theory, Series A, 162:241-279, February 2019.

[8] Yuval Filmus, Guy Kindler, Elchanan Mossel, and Karl Wimmer. Invariance principle on the slice. In 31st Computational Complexity Conference, 2016.

[9] Yuval Filmus and Elchanan Mossel. Harmonicity and invariance on slices of the Boolean cube. Probability Theory and Related Fields, to appear.

[10] Yuval Filmus, Ryan O'Donnell, and Xinyu Wu. A log-Sobolev inequality for the multislice, with applications. In Proceedings of the 10th Innovations in Theoretical Computer Science conference (ITCS'19), 2019.

[11] Nathan Keller and Ohad Klein. Kindler-Safra theorem for the slice. Manuscript, 2017.

[12] Tzong-Yau Lee and Horng-Tzer Yau. Logarithmic Sobolev inequality for some models of random walks. Ann. Prob., 26(4):1855-1873, 1998.

[13] Marvin L. Minsky and Seymour A. Papert. Perceptrons: Expanded Edition. MIT Press, Cambridge, MA, USA, 1988.

[14] Noam Nisan and Mario Szegedy. On the degree of Boolean functions as real polynomials. Comp. Comp., 4(4):301-313, 1994.

[15] Ryan O’Donnell. Analysis of Boolean functions. Cambridge University Press, 2014.

[16] Jake Wellens. A tighter bound on the number of relevant variables in a bounded degree Boolean function. CoRR, abs/1903.08214, 2019. 
[17] Karl Wimmer. Low influence functions over slices of the Boolean hypercube depend on few coordinates. In $C C C, 2014$. 